This is the final peer-reviewed accepted manuscript of:

Làdavas, E., Giulietti, S., Avenanti, A., Bertini, C., Lorenzini, E., Quinquinio, C., \& Serino, A. (2015). A-tDCS on the ipsilesional parietal cortex boosts the effects of prism adaptation treatment in neglect. Restorative Neurology and Neuroscience, 33(5), 647-662.

The final published version is available online at:

https://doi.org/10.3233/RNN-140464

Rights / License:

The terms and conditions for the reuse of this version of the manuscript are specified in the publishing policy. For all terms of use and more information see the publisher's website.

This item was downloaded from IRIS Università di Bologna (https://cris.unibo.it/)

When citing, please refer to the published version. 


\title{
a-tDCS on the ipsilesional parietal cortex boosts the effects of prism adaptation treatment in neglect
}

\author{
Elisabetta Làdavas ${ }^{\mathrm{a}, \mathrm{b}}, *$, Sara Giulietti ${ }^{\mathrm{c}}$, Alessio Avenanti $^{\mathrm{a}, \mathrm{b}, \mathrm{c}}$, Caterina Bertini $^{\mathrm{a}, \mathrm{b}}$, Eleonora Lorenzini ${ }^{\mathrm{c}}$, \\ Cristina Quinquinio ${ }^{\mathrm{d}}$ and Andrea Serino ${ }^{\mathrm{a}, \mathrm{b}, *, 1}$
}

${ }^{\mathrm{a}}$ Dipartimento di Psicologia, Università degli Studi di Bologna, Italy

${ }^{\mathrm{b}}$ Centro studi e ricerche in Neuroscienze Cognitive, Campus di Cesena, Cesena, Italy

'IRCCS Fondazione Santa Lucia, Roma, Italy

${ }^{\mathrm{d}}$ Istituto di Riabilitazione Santo Stefano, Porto Potenza Picena, Macerata, Italy

1Present address: Center for Neuroprosthetics, Ecole Polytechnique Federale de Lausanne, Switzerland.

*Corresponding author: Elisabetta Làdavas, Centro studi e ricerche in Neuroscienze Cognitive, Viale Europa 980, 47123 Cesena, Italy. Tel.: +39 051 2091347; Fax: +39 051 2091844; E-mail: elisabetta.ladavas@ unibo.it and Andrea Serino, Center for Neuroprosthetics, Ecole Polytechnique Federale de Lausanne, Station 19, CH-1015, Lausanne, Switzerland. Tel.: +41 2169

30695; Fax: +41 2169 31770; E-mail: andrea.serino@epfl.ch.

\begin{abstract}
.
Background and objective: The aim of the study is to compare the effects of multiple sessions of cathodal (c-tDCS) or anodal tDCS (a-tDCS) in modulating the beneficial effects of prism adaptation (PA) treatment in neglect patients. Methods: 30 neglect patients were submitted to 10 daily sessions of PA treatment. Patients were pseudo-randomly divided into 3 groups. In the c-tDCS-group, each PA session was coupled with 20 minutes of cathodal stimulation of the left, intact PPC; in the a-tDCS-group, anodal stimulation was applied to PPC of the damaged hemisphere; in the Sham group, sham stimulation was applied. Neglect was evaluated before and after treatment with the Behavioral Inattention Test.

Results: Combined tDCS-PA treatment induced stronger neglect improvement in the a-tDCS group as compared to the Sham group. No improvement was found in the c-tDCSgroup, with respect to that normally induced by PA and found in the Sham group.

Conclusions: c-tDCS abolished neglect amelioration after PA, possibly because stimulation affected the sensorimotor network controlling prism adaptation. Instead, a-tDCS PPC boosted neglect amelioration after PAprobably thanks to increased excitability of residual tissue in the lesioned hemisphere, which in turn might reduce dysfunctional overexcitability of the intact hemisphere.
\end{abstract}

Keywords: Neglect, Prism Adaptation, tDCS 



\section{Introduction}

Hemispatial neglect is a common outcome after right hemisphere stroke and is characterized by a deficit in perceiving, orienting, and moving toward the left side of space (Halligan et al., 1989). One proposal about the mechanisms contributing to neglect has invoked interhemispheric rivalry or competition (Kinsbourne, 1993). Because interhemispheric projections are believed to be inhibitory (Sprague, 1966), their loss, due to the impairment of one hemisphere, would result in a hyperexcitation of the intact hemisphere. As a result of a hyperactivation of the intact hemisphere, with concurrent hypoactivation of the damaged hemisphere, patients focus their attention on the ipsilesional field and the contralesional field lacks sufficient attentional resources. Stemming from this theoretical background, transcranial magnetic stimulation studies have shown that disturbing or inhibiting cortical functioning of the left posterior parietal cortex (PPC) is able to attenuate the pathological over-excitability of parieto-motor circuits in neglect patients, and ameliorate clinical aspects of neglect (Oliveri et al., 2001; Brighina et al., 2003; Shindo et al., 2006; Koch et al., 2008, 2012; Nyffeler et al., 2009; Song et al., 2009; Lim et al., 2010). These findings provide physiological and behavioral evidence for the idea that unbalanced excitability of the two hemispheres is an important contributor to neglect. If this theory of imbalance is correct, restoration should be conceived as a rebalancing act. This can be done by inhibiting the overexcited intact tissue and/or increasing excitability of the residual tissue in the ipsilesional cortex (Fregni et al., 2005; Hummel and Cohen, 2006; Boggio et al., 2007; Talelli and Rothwell, 2006; Hummel et al., 2008; Avenanti et al., 2012b; Mylius et al., 2012). A recent technique which has been safely applied to change patterns of hemispheric activation and inhibition in brain damaged patients is transcranial direct current stimulation (tDCS) (Ko et al., 2008; Feng et al., 2013; Sparing et al., 2009). tDCS is based on the application of low-amplitude electric current (typically 1-2 mA) to the scalp through relatively large sponge-based electrodes (e.g. $35 \mathrm{~cm} 2$ ). tDCS has been shown to elicit polarity-dependent excitability changes in the cortical area under the stimulation electrodes. Studies in the motor cortex showed that anodal tDCS (a-tDCS) increases motor excitability while cathodal tDCS (c-tDCS) decreases it (Antal et al., 2004; Nitsche and Paulus, 2000; see Horvath et al., 2015 for a recent quantitative meta-analysis), although many factors may contribute to the efficacy of the stimulation, including intensity, electrode size and disposition and duration of stimulation (Nitsche and Paulus, 2000; Nitsche et al., 2008; Mylius et al., 2010). Importantly, similar polarity-dependent effects can be reliably observed at the behavior level, at least when testing perceptual/attentional cognitive functions (Jacobson et al., 2012), with a-tDCS and c-tDCS being involved in the enhancement and inhibition of such functions, respectively.

Brain stimulation protocols based on tDCS may represent an ideal tool to promote neural plasticity in stroke patients, because compared to other noninvasive techniques (i.e. TMS), tDCS is easy to use, and can induce longer-lasting and more diffuse effects on neural networks which may be preferable for therapeutic purposes. However, to date only a few studies have used tDCS to ameliorate symptoms of neglect in stroke patients. Two studies successfully applied single doses of tDCS to induce short-term improvements. In a crossover study on subacute patients, Ko and colleagues (Ko et al., 2008) first showed that a-tDCS (2 mA, $20 \mathrm{~min}$ ) over the right PPC improved performance in two neglect tests (figure cancellation and line bisection), while sham stimulation did not. In a cross-over study on a group composed by subacute and chronic patients, Sparing et al. (2009) demonstrated that both a-tDCS (1 mA, $10 \mathrm{~min})$ over the affected PPC or c-tDCS (1 mA, $10 \mathrm{~min})$ over the intact homolog area reduced symptoms of neglect, as assessed by a line bisection task and the subtest for neglect from an attention test battery. More recently, bilateral tDCS (a-tDCS over the affected PPC and ctDCS over the intact PPC; daily sessions of 20 min at $1 \mathrm{~mA}$ intensity) was applied simultaneously with a computerized neuropsychological training program for neglect in a sham-controlled single case study (Brem et al., 2014). Results suggested that the simultaneous coupling of tDCS and standard neuropsychological training is feasible and tDCS may enhance training-induced improvements in measures of visuospatial neglect. However, two outstanding issues remain to be addressed. First, it is critical to provide group-level evidence that the neuromodulatory effect of tDCS could boost the effect of neuropsychological treatment of neglect. Second, although previous research has demonstrated beneficial effects of single-doses of a-tDCS and c-tDCS on neglect patients (Ko et al., 2008; Sparing et al., 2009), it is unclear which stimulation strategy is more effective in modulating the outcomes of neuropsychological rehabilitation in such patients.

A variety of rehabilitation techniques for neglect have been explored (see e.g., Cappa et al., 2005: Bowen et al., 2007 for review) and the prism adaptation technique (PA), in particular, has been shown to ameliorate neglect symptoms in large samples of patients (see Luauté et al., 2006a; Chokron et al., 2007; Barrett et al., 2012 for reviews). PA requires the patient to perform a series of pointing movements (with the ipsilesional hand) toward a visual target while wearing prismatic goggles. These goggles induce a deviation of the visual 
field toward the right. To compensate, the patient has to orient the pointing movement toward the left, resulting in a leftward drift of sensorimotor coordinates, both during exposure to prism (i.e. error reduction) and after prism removal (after-effect). Several studies have shown that this sensorimotor adaptation improves most neglect symptoms both for a short period of time after a single session of PA (Rossetti et al., 1998; Farnè et al., 2002) and in the long term, up to 6 months, after 2 weeks of daily treatment (Frassinetti et al., 2002; Serino et al., 2006, 2007, 2009; Làdavas et al., 2011).

In the present research, we sought to investigate whether the multiple sessions of c-tDCS applied over the left PPC in the intact hemisphere or a-tDCS applied over the right PPC in the lesioned hemisphere, might boost the beneficial effects of PA. A total of 19 post-acute neglect patients after right stroke performed PA treatment, in combination with tDCS protocols that were effective in enhancing (a-tDCS) or inhibiting (c-tDCS) parietal functioning both in neurologically intact individuals and stroke patients (Ko et al., 2008; Bolognini et al., 2013, 2015; Convento et al., 2014; Minamoto et al., 2014). Eleven patients were tested in the a-tDCS group and eight patients were tested in the c-tDCS group. To potentially prime functional networks and influence neuropsychological treatment, tDCS was applied before and partially during each of 10 daily sessions of PA. Results were then compared with those obtained in a control condition, in which PA treatment was associated with sham tDCS, run in a different group of neglect patients $(N=11)$. For each of the three groups, PA treatment was applied in 10 sessions over 2 weeks. The severity of neglect was assessed before and after the combined treatment through the Conventional Battery of the Behavioral Inattention Test (BIT; Wilson et al., 1987), a comprehensive battery that consists of a number of standardized tests, such as line crossing, letter cancellation, star cancellation, figure and shape copying, line bisection, and drawing. If tDCS effectively augments rehabilitation outcomes, in line with the imbalance theory whereby restoration is due to a rebalancing act, stronger neglect improvement should be observed after active tDCS conditions than after sham tDCS. The comparison between the results obtained after ctDCS over the left PPC and a-tDCS over the right PPC would thereafter indicate which strategy is optimal for enhancing the effect of PA. Importantly, the outcome of the treatment might depend on the neural networks underlying the mechanisms of prism adaptation. If PA is at least partially mediated by parietal sensorimotor networks in the left, intact hemisphere, contralateral to the hand performing the pointing movements (Luauté et al., 2006, 2009), one may expect little or no improvement after c-tDCS over left PPC. Indeed, while such brain stimulation protocol might ameliorate neglect symptoms when uncoupled with a specific treatment (Sparing et al., 2009), it can also interfere with motor control functions of parietal regions (Convento et al., 2014) and thus with the PArehabilitation itself. Rather, a greater improvement is expected after a-tDCS over right PPC, as anodal stimulation of PPC has been proven to enhance parietal functions (Convento et al., 2014; Minamoto et al., 2014; Bolognini et al., 2015) and improve neglect symptoms (Ko et al., 2008). AtDCS over right PPC may activate residual tissue in the affected parietal cortex, without interfering with left hemisphere sensorimotor networks mediating the progressive realignment process, necessary for PA to occur. Therefore, greater neglect improvement can be expected in the a-tDCS group relative to the others two groups.

\section{Method and procedure}

\section{Design and patients selection}

This is a 3 parallel groups, double-blinded study, with imbalanced randomization (see below). Thirty righthanded patients presenting with neglect after right hemisphere stroke were recruited to participate in the study at the Istituto di Riabilitazione Santo Stefano, MC, Italy, between January 2008 and June 2012 (Table 1). From a sample of 92 stroke patients, with unilateral right brain damage, admitted to the Institute, patients with hemispatial neglect, meeting the following criteria, were selected; the inclusion criterion was their pathological performance on the Behavioral Inattention Test (BIT) battery (i.e., with BIT Conventional scores $\leq 129$ (see below). Exclusion criteria were the presence of widespread mental deterioration (Mini-Mental State Examination score <20), psychiatric disorders, a history of prior stroke or prior haemorrhage, any severe internal medical disease, epilepsy and additional factors influencing the risk of epilepsy (i.e. assumption of medications that raise the seizure threshold, metal in the head, alcohol consumption, excessive caffeine intake). In addition, all patients were submitted to prolonged EEG monitoring to exclude presence of epileptic activity before inclusion in the study. All patients gave informed consent for participation in the study. Experimental procedures were approved by the ethics committees of Istituto di Riabilitazione Santo Stefano and of 
Departmentof Psychology, University of Bologna (Ref: N.148, 3.5.11) and conducted in accordance with the Declaration of Helsinki.

Power analyses were conducted on the basis of the effect size of PA treatment reported in 3 previous papers (Serino et al., 2006, 2007, 2009) using the same procedure of PA as in the present study, on a total of 45 patients (average BIT-C scores \pm s.e.m.: Baseline $=99.25 \pm 6.6$; post-treatment $=125.57 \pm 6.4$; effect size dz $=1.21$ ). Results indicated an adequate sample size of 10 subjects per group for a one-sided $5 \%$ significance level; therefore, we included a total of 30 patients in the study. A two-steps approach was used to assign patients to the different experimental groups. Twenty-four patients ( 8 per group) were initially randomly assigned to sham tDCS, c-tDCS over the contralesional PPC or to a-tDCS over the ipsilesional PPC. From each triplet of patients, the first patient was assigned to the sham group, the second to the c-tDCS group, and the last one to the a-tDCS group, with the order of group assignment varying between patients' triplets. At the end of this stage of the research, an interim analysiswas conducted on data from 8 patients per group. No improvement after PA was shown in patients assigned to the c-tDCS group. Thus, for ethical reasons, and as required from the ethical committee of the Istituto di Riabilitazione Santo Stefano, no more patients were assigned to this procedure (final $N=8$ ). Thereafter, the next 6 recruited patients were assigned to either the sham PPC tDCS (final $N=11$ ) or to the real anodal tDCS (final $N=11$ ) condition. The groups did not differ between each other in gender (all $p$ values $=1$ ), mean age (all $p$ values $>0.16$ ), education (all $p$ values $>0.24$ ), duration of illness (all $p$ values $>0.27$ ), or baseline BIT Conventional scores (all $p$ values $>0.56$ ), and had similar stroke location (Table 1). Patients were unaware of their group assignment; all were only told that they had been enrolled in rehabilitation treatment for their spatial attention deficits (involving a neuromodulator session with tDCS and a training session with PA). The neuropsychologist who performed neglect evaluation before and after the treatment was blind with respect to the patients' group assignment. The researcher who generated the patients' allocation sequence did not perform the neuropsychological evaluation, nor administered the treatment.

\section{PA procedure}

The 2-week rehabilitation program consisted of 10 sessions lasting 30 minutes each and held 5 days per week. During PA treatment, patients were required to repeatedly point at a visual target with their right index finger while wearing prismatic lenses, which shifted their visual field $10^{\circ}$ rightward.Visual targets were presented 90 times, $60 \mathrm{~cm}$ away from the patient's midline subtending a total visual space of 50 . The visual targets were presented 30 times in a variety of positions in the patient's right visual field (randomly chosen over a range between +25 and $+15 \mathrm{deg}$ ), 30 times in the left (randomly chosen over a range between -25 and $-15 \mathrm{deg}$ ) and 30 times in the center (randomly chosen over a range between +5 and $-5 \mathrm{deg}$ ), in random order. During the adaptation procedure, the pointing movement was hidden below the top face of a custom-made structure (see Serino et al., 2006, 2007, 2009; Làdavas et al., 2011) until the final part of the movement (approximately the last $12 \mathrm{~cm}$ ), when the index finger emerged beyond the distal edge of the structure. In this way, patients could only see the final part of their movement, i.e., their index finger, thus enabling them to progressively correct the pointing errors induced by the prism. This amount of visual feedback has been proved to be more effecting in recovering neglect visuo-spatial deficits (see Làdavas et al., 2011).

\section{Transcranial direct current stimulation}

tDCS was applied using a battery-driven Eldith (neuroConn GmbH, Ilmenau, Germany) Programmable Direct Current Stimulator with a pair of surface saline-soaked sponge electrodes $(5 \times 7=35 \mathrm{~cm} 2)$. Rubber bandages were used to hold the electrodes in place for the duration of stimulation. In each session, we delivered a constant current of $2 \mathrm{~mA}$ intensity (current density: $0.57 \mathrm{~mA} / \mathrm{cm} 2$ ) complying with current safety data (Nitsche et al., 2003a; Poreisz et al., 2007). Stimulation lasted for a total of $20 \mathrm{~min}$ not including $20 \mathrm{~s}$ of ramp up and ramp down at the beginning and end of stimulation. Impedance was constantly monitored and kept below 8 $\mathrm{kOhm}$.

Two different electrode montages targeted the PPC in the left and right hemispheres using the P5 and P6 positions of the international 10-20 system for EEG electrode placement (Fierro et al., 2000, 2001; Oliveri et al., 2001; Brighina et al., 2003; Shindo et al., 2006; Lim et al., 2010). More specifically, for a-tDCS, the anodal electrode was placed over P6 (right PPC), while the reference electrode was applied over the left supraorbital region. We selected this arrangement of the reference and stimulation parameters ( $2 \mathrm{~mA}$ for $20 \mathrm{~min}$ ) based on 
the study of Ko and colleagues (2008), who showed that a single-dose of such a-tDCS protocol was effective in ameliorating neglect symptoms. Moreover, a-tDCS with similar PPC-contralateral orbit montage and stimulation intensity enhanced parietal functioning (e.g. performance in attentional tasks, action planning) in other studies on healthy subjects (Convento et al., 2014; Minamoto et al., 2014) and stroke patients (Bolognini et al., 2015). For the c-tDCS, the cathodal electrode was placed over P5 (left PPC) and the reference electrode was positioned over the right supraorbital region. This PPCcontralateral orbit montage at an intensity of $2 \mathrm{~mA}$ was proven to inhibit parietal functioning in previous studies (Bolognini et al., 2013; Convento et al., 2014; Minamoto et al., 2014).

The montage used in the sham group mimicked that used in the two active groups: in 5 patients, the cathode was placed over P5 and anode over the right supraorbital region, in the remaining 6 patients, the anode was placed over P6 and the cathode over the left supraorbital region. The sham protocol ensured that subjects would feel the initial itching sensation at the beginning of tDCS; electrodes were placed in the same positions as PPC tDCS, but the stimulator was turned off after just $30 \mathrm{~s}$ (fade in/out: $20 \mathrm{~s}$ ), preventing any effective modulation of cortical excitability. This procedure ensures successful blinding of participants when using lower tDCS intensities (1 mA) (Gandiga et al., 2006; Ambrus et al., 2012) whereas it may be less effective at the intensity used in our study ( $2 \mathrm{~mA}$ ) (O'Connell et al., 2012). However, the risk of unblinding should be minor considering that we did not use a cross-over design and all patients were naive to tDCS (see Loo et al., 2010, 2012 for evidence of effective blinding using similar stimulation parameters). Adverse events were monitored by asking patients after each session of stimulation and in the follow-up if they have experienced any adverse event and the relationship of these events to the treatment with tDCS.

Although it is still not clear which is the optimal way of combining tDCS and neuropsychological training, clinical trials have shown beneficial effects when applying tDCS at the onset of the training (Baker et al., 2010; Kim et al., 2010; Lindenberg et al., 2010; Bolognini et al., 2011; Marangolo et al., 2011) or some minutes earlier, to assure that training was performed under stimulation (Fridriksson et al., 2011; Brem et al., 2014). In these studies, tDCS was administered during the entire duration of the training or, in most of the cases, its initial part (i.e. the training typically lasted some minutes/hours longer than tDCS). Other studies, using tDCS or TMS have also suggested that brain stimulation applied before training induce training-dependent enhancements (Koganemaru et al., 2010; Avenanti et al., 2012b; Giacobbe et al., 2013) suggesting the beneficial effect of priming functional network prior to learning. In this study, tDCS lasted for 20 min. PA training started 10 min after the onset of the stimulation (we reasoned that this timing was enough for tDCS to induce changes in excitability in the target networks, see Nitsche and Paulus 2000, 2001; Nitsche et al., 2003a, 2003b; Ardolino et al., 2005) and lasted $30 \mathrm{~min}$. During the first $10 \mathrm{~min}$ of stimulation, patients were at rest, whereas during the following 10 min they started to perform the PA treatment.

\section{Neglect assessment}

In order to provide a general, clinically relevant measure of neglect deficits and improvement, the outcome of the treatment was evaluated by using the Conventional Battery from the Behavioural Inattention Test (BIT), with scores up to 129 considered as indicative of neglect (range: 0-146). Neuropsychological evaluation was performed by blinded raters before the treatment, within the week preceding the first session of PAtreatment, and after the treatment, within the first week after the last session of PA treatment.

\section{Lesion analysis}

Patients' brain lesions were identified in the MRI scans by an experienced neuropsychologist for all patients but one (Case P4 from Sham group for whom only the medical report of the original CT scan was available). Brain lesions were drawn onto a normalized MNI template (www.bic.mni.mcgill.ca/cgi/icbm view) using MRIcro (www.mricro.com; Rorden and Brett, 2000). Subsequently, the locations of the lesions were identified using the Automated Anatomical Labelling map (Tzourio-Mazoyer et al., 2002) provided by the software, and with reference to the atlas of Duvernoy et al. (1991). Lesion reconstructions are reported in Fig. 3.

\section{Results}

Patients reported no significant adverse effect of tDCS, except only a few cases of minimal irritation of the skin beneath the electrodes. An ANOVA run on BIT scores with the factors Session (Pre- and Post-training) and Group (Sham, a-tDCS and c-tDCS) revealed a main effect of Session $[\mathrm{F}(1,27)=80.25, p<0.001 ; \eta 2=$ 0.42], with higher scores after the treatment (mean score \pm s.e.m.: $112 \pm 6)$ than before the treatment $(101 \pm$ 
5), showing a general beneficial effect of PA on neglect recovery. More importantly, the Session $\times$ Group interaction was also significant $[F(2,27)=4.06 ; p<0.05 ; \eta 2=0.23]$. Pre and posttraining results are reported in Fig. 1. Newman-Keuls post-hoc comparisons showed that the patients from both the Sham and the a-tDCS groups significantly improved after the training (Sham $=112 \pm 9$; a-tDCS $=120 \pm 4$ ) as compared to before the training (Sham $=103 \pm 10$; a-tDCS $=101 \pm 8$; both $\mathrm{p}<0.05 ; \eta 2=0.52$ and 0.56 , respectively). In contrast, no improvement was observed in patients from the c-tDCS group in the post-training (101 \pm 16$)$ compared to the pre-training $(99 \pm 15)$ sessions $(p=0.78 ; \eta 2=0.16)$. Moreover, there was no difference in the performance by the 3 groups in the pre-training session (all $\mathrm{p}$ values $>0.61$ ), whereas post-training scores were significantly higher in the Sham and in the a-tDCS group compared to the c-tDCS group (both $p$ values $<0.05$ ), and also in the a-tDCS group compared to the Sham group $(p<0.05)$. The main effect of Group was not significant $(p=$ $0.77 ; \eta 2=0.019)$.

In addition to traditional mixed ANOVA, in line with recent suggestions in the field (Goedert et al., 2013), we also run a statistical analysis based on Mixed Linear Modeling(MLM).MLM, indeed, better accounts for between-subject heterogeneity in neglect severity and in recovery, and does not make rigid assumption regarding the data structures.To this aim, BIT scores were entered as dependent variable, Group (Sham, atDCS, c-tDCS) and Session (Pre-, Post-Treatment) as factorial fixed effects and participants' intercepts were modeled as a random effect. The MLM analysis revealed a main effect of Session $(\mathrm{F}(1,27)=19.4 ; p<0.0001)$ and a significant Session X Group interaction $(\mathrm{F}(2,72)=4.02 ; p=0.03)$, and no main effect of Group $(p=$ 0.71), thus replicating the results from the mixed ANOVA described above. In order to test whether anodal tDCS boosted the PA effects as compared to the improvement induced by PA alone in the Sham group, we also run MLM analyses on BIT scores post-training, with patients as random factor, group including only sham and a-tDCS group, as fixed factor, and BIT scores pre-training as a covariate factor. Results showed a significant effect of Group $(p=0.01)$ and a significant BIT pre-training $\times$ Group interaction $(p=0.004)$, showing that a significant increase in neglect amelioration after the coupling of PA and a-tDCS can be demonstrated, as compared to coupling PA with sham stimulation, when inter-individual variability and patients' level before the treatment are taken into account. Improvements by individual patients from the 3 groups is also reported in Fig. 2.

\section{Lesion analyses}

The location and the extent of patients' brain lesion are shown in Fig. 3

A first lesion analysis was run in order to check that the differential effect on neglect recovery after combined tDCS and PA treatment for Sham, a-tDCS and c-tDCS patients was not due to a different distribution of brain damage in the 3 groups as a consequence of an uncontrolled group assignment bias. To this aim, we firstly compared the general extent of damaged tissue between the 3 groups. For each patient, we calculated the proportion of general brain damaged, by computing and summing the proportion of damaged voxels in each brain area considered by the Automated Anatomical Labelling map (Tzourio-Mazoyer et al., 2002) embedded in MRIcro. Those indexes were compared between the 3 groups by means of a one-way between-subjects ANOVA. There was no group difference in the general proportion of brain damage $(p=0.71$; Mean proportion of damaged voxels \pm s.e.m.: Sham $=13 \% \pm 6$; a-tDCS $=9 \% \pm 2$; c-tDCS $=14 \% \pm 3$ ).

In addition, we controlled that the extent of brain damage in those areas where tDCS was applied was balanced between the 3 groups. To this aim, we calculated the proportion of damaged voxels in posterior parietal regions, approximately corresponding to the location of P6 electrode (Fierro et al., 2000, 2001; Oliveri et al., 2001), used as target position for the anodal electrode in the a-tDCS group. As it is well known that effects of tDCS are not focal, we considered the following regions of the right PPC: the angular gyrus, the supramarginal gyrus, the inferior parietal lobule and the superior parietal lobule that were likely affected by anodal current. No analysis was conducted for brain lesions at the location of P5 electrode, used as a reference for the cathodal electrode in c-tDCS patients, because none of the patients presented a lesion in the left hemisphere. The proportion of damaged voxels in the right angular gyrus, supramarginal gyrus, inferior parietal lobule and superior parietal lobule was compared between the 3 groups with a series of between-subjects one-way ANOVAs. The extent of the brain lesion was not different between the 3 groups in the angular gyrus $(p=0.71$; Mean proportion of damaged voxels \pm s.e.m.: Sham $=22 \% \pm 10$; a-tDCS $=13 \% \pm 9$; $\operatorname{ctDCS}=13 \% \pm 10$ ), the supramarginal gyrus $(\mathrm{p}=0.85$; Sham $=29 \% \pm 12$; $\mathrm{a}-\mathrm{tDCS}=23 \% \pm 10 ; \mathrm{c}-\mathrm{tDCS}=32 \% \pm 13)$, the inferior 
parietal lobule $(\mathrm{p}=0.93$; Sham $=16 \% \pm 11 ; \mathrm{a}-\mathrm{tDCS}=11 \% \pm 9$; $\mathrm{c}-\mathrm{tDCS}=11 \% \pm 11)$ and the superior parietal lobule $(\mathrm{p}=0.57$; Sham $=11 \% \pm 9 ; \mathrm{a}-\mathrm{tDCS}=8 \% \pm 5 ; \mathrm{ctDCS}=2 \% \pm 2)$.

Theoretical arguments have suggested that excitatory brain stimulation protocols over lesioned areas may be problematic because: i) damaged tissue is more epileptogenic and thus its stimulation is potentially risky; ii) the stroke scar may shunt the induced currents and lead to mislocalization of the stimulation and thus the effect of such excitatory protocols may be of difficult interpretation (Hummel et al., 2008; Adeyemo et al., 2012). Although available data seem not to support point i) (Mylius et al., 2012; Ayache et al., 2012), it is reasonable to apply a-tDCS over relatively spared cortical areas to have clear neuromodulatory effects and minimize any risk. Hence, we checked that no patient in the a-tDCS group had complete damage in the right PPC region corresponding to the position of the anodal electrode. With the exception of two patients (P6 with 81\% and P4 with $38 \%$ of damaged voxels), all participants in the a-tDCS had small lesions in the target area $(<20 \%$ of damaged voxels).

We also checked whether the 3 groups were matched for the extent of frontal, temporal, parietal, occipital and subcortical (namely in the putamen, caudatum, pallidum and thalamus) damage. To this aim, we computed the number of damaged voxels in each area indicated by the Anatomical Labelling map in MRIcro, we summed those values for the different areas belonging to each region, and we extracted the proportion of damaged tissue for each region. Those indexes were compared between the 3 groups by means of series of one-way ANOVAs with the between-subjects factor of group. There was no between group difference for any of the considered region. (Frontal areas: $\mathrm{p}=0.19$; Sham $=11 \% \pm 9$; a-tDCS $=7 \% \pm 3$; c-tDCS $=9 \% \pm 3$; Temporal areas: $\mathrm{p}=$ 0.33 ; Sham $=14 \% \pm 5$; a-tDCS $=9 \% \pm 3$; c-tDCS $=17 \% \pm 5$; Parietal areas: $\mathrm{p}=0.84$; Sham $=14 \% \pm 8$; a$\mathrm{tDCS}=10 \% \pm 5 ; \mathrm{ctDCS}=10 \% \pm 5$; Occipital areas: $\mathrm{p}=0.66$; Sham $=4 \% \pm 3$; a-tDCS $=2 \% \pm 2 ; \mathrm{c}-\mathrm{tDCS}=$ $1 \% \pm 1$; Subcortical areas: $\mathrm{p}=0.43$; Sham $=9 \% \pm 7$; a-tDCS $=12 \% \pm 3$; $\mathrm{c}$-tDCS $=19 \% \pm 6$ ).

Finally, we investigated whether the different extension of lesions across the whole brain or in the different brain regions could partially explain the differential effects of combined tDCS-PA treatment in the three groups. To this aim, we firstly run an ANCOVA on the BIT scores data, with the factor Session (pre-, posttreatment) and Group (Sham, a-tDCS, c-tDCS), and with the total proportion of damaged voxels as a covariate. Results confirmed the significant effect of Session $(\mathrm{F}(1,25)=14.71 ; p<0.0001)$ and, more importantly, the significant two-way Session $\times$ Group interaction $(\mathrm{F}(2,21)=3.46 ; p<0.05)$, with no significant further effect of general proportion of damaged voxels, nor any interaction between brain damage and the other factors (all $p$ values $>0.32$ ). In addition, we also tested whether the location of brain lesions could, at least partially, explain neglect amelioration after different combinations of tDCS and PA. We run an ANCOVA on the BIT scores data, with the factor Session (pre-, posttreatment) and Group (Sham, a-tDCS, c-tDCS), and with the proportion of damaged voxels in the frontal, temporal, parietal, occipital and subcortical regions as covariates. Results confirmed the significant two-way Session $\times$ Group interaction $(F(2,21)=3.78 ; p<0.05)$, with no significant further effect of the brain damage, nor interaction between brain damage and the other factors (all $p$ values $>0.30$ ).

\section{Discussion}

The aim of the present study was to test whether and how the neuromodulatory effect of tDCS could be used to boost effectiveness of PA treatment in neglect patients. To this aim, we compared the effects of pairing PA with the c-tDCS applied over PPC in the left, intact hemisphere and the a-tDCS applied over PPC in the lesioned, right hemisphere, with those obtained in a control condition, in which PA was paired with a sham tDCS.

The results show that in the sham group the repetition of pointing movements toward visual stimuli, while the patient was wearing the prism, improved visuospatial performance. The exercise requires the patient to plan and perform a series of movements toward a visual stimulus presented in different positions of the visual field and to compensate for the visual displacement induced by prisms. Since pointing relies on a form of visuomotor coordination between the hand and eye (Neggers and Bekkering, 2000), pointing exercises during PA train the patient to re-orient the eye-hand sensorimotor system toward the left side of space in order to compensate for the right displacement induced by prisms (Angeli et al., 2004a, 2004b; Serino et al., 2006). The beneficial effect of prism adaptation on neglect has already been demonstrated in a number of previous studies (Rossetti et al., 1998; Frassinetti et al., 2002; Serino et al., 2006, 2007, 2009: Vangkilde and Habekost, 2010; Fortis et al., 2010; Làdavas et al., 2011) and PA is considered to be one of the best tools for treating neglect at the 
present moment (see Zoccolotti et al., 2011; Barrett et al., 2012). However, some trials failed in reporting statistically significant long term improvements after PA (Turton et al., 2010; Nys et al., 2008) and not all patients treated with PA fully benefited from the treatment (see Barrett et al., 2012 for comments). Therefore, novel adjuvant treatments potentially capable of enhancing the benefits of PA are highly desirable.

The important new finding from the present study is that the beneficial effects of PA were significantly boosted if the treatment was coupled with a-tDCS applied over the affected right PPC. In contrast, when PA treatment was coupled with c-tDCS applied over the unlesioned PPC, no improvement at all was observed. Previous studies have shown that upregulating excitability in the affected PPC or inhibiting the intact PPC may ameliorate neglect symptoms in stroke patients (see e.g., Oliveri et al., 2001; Brighina et al., 2003; Shindo et al., 2006; Koch et al., 2008, 2012; Ko et al., 2008; Sparing et al., 2009; Nyffler et al., 2009; Song et al., 2009; Lim et al., 2010) and in general this approach has found support also in other domains, as for example in the motor domain, whereby improvements of motor dexterity in hemiparetics has been reported both after excitatory stimulation of the affected motor cortex or inhibitory stimulation of the unaffected motor cortex (Khedr et al., 2005, 2009; Mansur et al., 2005; Takeuchi et al., 2005; Fregni et al., 2006; Kim et al., 2006; Boggio et al., 2007; Ameli et al. 2009).

Relevant for the present study is the work of Ko and colleagues (2008) who used a-tDCS over the right PPC ( $2 \mathrm{~mA}$ for $20 \mathrm{~min}$ ) and found short-term improvements in neglect symptoms. Additionally, Sparing et al. (2009) showed that both c-tDCS over the left PPC and a-tDCS over the right PPC reduced neglect symptoms in stroke patients. Our a-tDCS results confirm and extend these previous findings, by showing that anodal currents over PPC applied before and partially during PA may result in a better functional outcome than PA combined with sham stimulation.

The a-tDCS protocol is thought to increase the excitability of the targeted network by acting on two possible mechanisms. When applied online a-tDCS is thought to induce effects that are related to membrane depolarization because they are affected by ion-channel blocking substances (Stagg and Nitsche, 2011), whereas off-line induced effects (i.e. effects that persist beyond the stimulation period) involve the additional participation of glutamatergic N-methyl-Daspartic (NMDA) receptors and therefore a long-term potentiationlike (LTP) mechanism (Liebetanz et al., 2002; Stagg and Nitsche, 2011). It is likely that both mechanisms influenced changes in behavior in the a-tDCS group, since our PA treatment started after 10 min stimulation a timing sufficient to induce longlasting increase of excitability for up to 1 hour (Nitsche and Paulus, 2001; Nitsche et al., 2003b; Ardolino et al., 2005) and was continued for 10 min during online stimulation (see Fridriksson et al., 2011; Brem et al., 2014 for similar approach in stroke patients). While the present study demonstrates a beneficial effect of applying a-tDCS over the right PPC before and partially during PA treatment, future studies are needed to clarify whether it is more promising to affect both

off-line and on-line a-tDCS mechanisms, or selectively focus on either of the two.

Our findings strongly support the notion that a-tDCS may improve neglect symptoms, however, they are in apparent contrast with previous research relative to the effect of c-tDCS. Indeed, the lack of improvement found in our c-tDCS group contrasts with the findings of Sparing and colleagues (2009) and with those of previous TMS studies showing beneficial effects of inhibiting the left PPC in stroke patients (Oliveri et al., 2001; Brighina et al., 2003; Shindo et al., 2006; Koch et al., 2008, 2012; Nyffler et al., 2009; Song et al., 2009; Lim et al., 2010).

One possible concern is that our c-tDCS protocol was not effective in inhibiting the intact PPC. Indeed, a previous study using $2 \mathrm{~mA}$ for 20 min over the motor cortex has suggested that this type of stimulation, with relatively high intensity and long duration, can be excitatory (Batsikadze et al., 2013; but see Tremblay et al., 2013 and Horvath et al., 2015). The study of Batsikadze et al. (2013) suggests that enhanced intensity and duration of c-tDCS may shift the direction of the changes in excitability, possibly because of an increase of calcium level to an amount that induces LTP-like plasticity.

However, it should be considered that in our study the distance between the target and reference electrode was greater compared to that study. Longer distances between electrodes might be associated with decreased stimulation efficiency (Moliadze et al., 2010), and this factor may have prevented our stimulation to become excitatory. Indeed, if the effects of our c-tDCS were excitatory, one should have expected an exacerbation of the neglect symptoms because of the over-activation of the left PPC in the intact hemisphere. In contrast, we did not find a worsening of neglect symptoms in the c-tDCS group, but rather no improvement after stimulation and PA treatment. Additionally, previous studies testing very similar stimulation and electrode arrangement over PPC found results compatible with a reduction of cortical excitability in the stimulated PPC (Bolognini et al., 2013; Convento et al., 2014; Minamoto et al., 2014). Interestingly, such effects were reported both when c-tDCS was applied online (Minamoto et al., 2014), and thus likely reflected a reduction in cortical excitability 
induced by membrane hyperpolarization, and when c-tDCS was administered offline (Bolognini et al., 2013; Convento et al., 2014), and thus likely acted on long term depression-like (LTD) mechanisms (Nitsche et al., 2008).

Thus, it appears rather that our c-tDCS protocol interfered with the effects of PA. In view of the temporal relation between the two interventions, it may be possible that either membrane hyperpolarization or LTD-like mechanisms (or both) may have negatively influenced learning during PA. In this vein, findings from the present study suggest that some principles of neuromodulation (i.e. inhibiting the healthy hemisphere) are not necessarily beneficial when coupled with another rehabilitation treatment, although it remains to understand whether online or offline influences (or their combinations as in the present study) may interfere most with treatment. Thus, our data suggest caution in designing coupled tDCS - rehabilitation interventions: when noninvasive brain stimulation is coupled with cognitive rehabilitation, it is critical to consider which neural mechanisms are recruited by the treatment. If these correspond to the networks that are affected by ctDCS, such stimulation can be detrimental rather than beneficial. This is probably what happened in the present study Few previous imaging studies investigated the anatomic substrates underlying the beneficial effect of PA in patients with persistent left neglect following right hemisphere stroke and have reported that beneficial effects of PA correlated with activation of the left PPC. In a first study, Luaute et al. (2006) used PET to examine the changes of regional cerebral blood flow as a function of neglect improvement. They found that regions associated with improvement of neglect produced by PA included right cerebellum, left thalamus, left temporooccipital cortex, left medial temporal cortex, and right posterior parietal cortex. Thus, PA seems to reduce neglect by facilitating the recruitment of an intact network of brain areas responsible for controlling normal visuospatial responses in the healthy hemisphere. In a further study (Luauté et al., 2009), run on healthy participants, the same group used an event-related fMRI design, which allows a dynamic investigation of neural activity over the time course of prism exposure, and found that the visuo-motor correction of pointing movements implemented during PA was related to an activation of the left PPC, i.e. in the left parieto-occipital sulcus (POS) and in left anterior intraparietal sulcus (antIPS). Similarly to neglect patients, participants in this study used their right hand during PA, and activation was related to the contralateral hemisphere. More specifically, it was found that the activity of POS was related to the reaching component and to the magnitude of error correction on the current trial. This activity was low in the first trials, where large terminal errors were present, and then progressively increased until errors were reduced (first 10 trials). In contrast, the left antIPS was activated according to pointing deviation, suggesting a role of this region in detecting pointing errors, which is consistent with a previous PET study reporting similar activation during a reaching task with jumping targets (Desmurget et al., 2001). Finally, the authors also found an activation in right intraparietal cortex during the early exposure phase, when comparing the first versus second runs of pointing with prisms and a bilateral activation of the superior temporal sulcus, associated with sustained exposure to prism. Activity increase in the right hemisphere was found only during the later stage, when pointing deviation was fully compensated and errors completely abolished.

Taken together, the aforementioned results support the view that the correction of sensory-motor coordinates is mediated by sensorimotor networks contralateral to the pointing hand and centered over the left PPC. In the case of neglect patients, if PA requires processes of error detection in the left anterior IPS and error correction in the left POS, it is not surprising that the inhibition of these neural structures by c-tDCS over the left PPC does not allow the adaptation to the visual disparity between proprioceptive and visual information and the consequent improvement of neglect deficits (Serino et al., 2006; Redding and Wallace, 2006). Our findings provide insights into the possible mechanisms underlying the recovery of neglect by using PA associated with tDCS. Our data indicate that c-tDCS over left PPC (in combination with a-tDCS over the right supraorbital regions), known to interfere with cortical functions of PPC (Bolognini et al., 2013; Convento et al., 2014; Minamoto et al., 2014), can disrupt the beneficial effect of PA when this tDCS protocol is coupled with PA treatment. This suggests that sensorimotor networks involving the left PPC are not only recruited during adaptation effects (Luauté et al., 2006, 2009), but migh be also necessary for the beneficial effect of PA (Saj et al., 2013). In addition, atDCS over right PPC (in combination with c-tDCS over the left supraorbital regions), known to enhance cortical functions of PPC (Bolognini et al., 2013; Convento et al., 2014; Minamoto et al., 2014), increases the beneficial effect of PA when this protocol is coupled with PA treatment, possibly by enhancing the recruitment of spared tissue in parietal regions.

Our study has some potential limitations, which require careful consideration. First, our stimulation had limited focality. Indeed, we used conventional bipolar electrode arrangement with large electrodes delivering a relatively nonfocal stimulation (Nitsche et al., 2008; Hummelet al., 2008). Additionally, our reference electrode was placed over the supraorbital region contralateral to the target electrode and therefore currents 
flowed between prefrontal and parietal regions in the two active stimulation groups. Although we selected this arrangement based on the evidence of clinical utility of a-tDCS in stroke patients (e.g. Ko et al. 2008; but see also more recent studies of Sunwoo et al., 2013 and Bolognini et al., 2015), future studies are needed to understand whether the observed effects are specific for the stimulation of left and right PPC, or may be mediated by larger parieto-frontal networks of sensorimotor regions connected to bilateral PPC. In this context, it should be considered however that studies using this montage have successfully modulated cortical functions that are typically ascribed to the parietal cortex (e.g. attention, planning phase of action performance; Minamoto et al., 2014; Convento et al., 2014; Bolognini et al., 2015), but not sensorimotor functions of and neural activity in frontal motor areas (Convento et al., 2014; Avenanti et al., 2012a). This suggests that neural effects of our montage might largely influence parietal (and possibly contralateral anterior prefrontal) areas, rather than frontal sensorimotor networks, suggesting at least partial anatomical specificity. While higher focality may be necessary to better understand the mechanisms of PA improvement future studies will also clarify whether more focal stimulations have some clinical utility. Indeed, it is held that recovery after a stroke strongly relies on the functional reorganization of large scale brain networks and thus recruitment of large neural networks is desirable in stroke rehabilitation.

In conclusion, the results of the present study suggest caution when coupling cognitive treatments with standard methods of brain stimulation aimed at re-balancing interhemispheric competition by downtuning the excitability of the contralesional hemisphere. Our data suggest that, it is important to understand which brain system mediates the recovery of the impaired function. If the neural networks underlying the recovery is implemented in the intact hemisphere, it can be predicted that its inhibitory stimulation can be detrimental. In contrast, for the reasons outlined above, the stimulation of the damaged hemisphere can be effective in boosting the recovery.

\section{Acknowledgments}

This study was funded by a MIUR-PRIN grant (Protocol: 2012E5FK8E-Attention and Perceptual Awareness) and by a FARB University of Bologna grant (Protocol, RFBO120993-Visual Awareness Lost and Found: Neural and Cognitive Mechanisms) to Elisabetta L'adavas and Andrea Serino, and by a scholarship from Istituto di Riabilitazione Santo Stefano to Sara Giulietti and Eleonora Lorenzini. The funders had no role in the design, conduction, analysis and writing of the study. We are grateful to Francesca Petrini for her help in lesion reconstruction. 


\section{References}

Adeyemo, B.O., Simis, M., Macea, D.D., \& Fregni, F. (2012). Systematic review of parameters of stimulation, clinical trial design characteristics, and motor outcomes in non-invasive brain stimulation in stroke. Front Psychiatry, 3, 88. doi: 10.3389/fpsyt.2012.00088

Ambrus, G.G., Al-Moyed, H., Chaieb, L., Sarp, L., Antal, A., \& Paulus, W. (2012). The fade-in-short stimulation-fade out approach to sham tDCS-reliable at $1 \mathrm{~mA}$ for naive and experienced subjects, but not investigators. Brain Stimul, 5(4), 499-504. 499-504

Ameli, M., Grefkes, C.,Kemper, F., Riegg, F.P., Rehme, A.K., Karbe, H., Fink, G.R., \& Nowak, D.A. (2009). Differential effects of high-frequency repetitive transcranial magnetic stimulation over ipsilesional primary motor cortex in cortical and subcortical middle cerebral artery stroke. Ann Neurol, 66(3), 298-309.

Angeli, V., Meneghello, F., Mattioli, F., \& L`adavas, E. (2004a). Mechanisms underlying visuo-spatial amelioration of neglect after prism adaptation. Cortex, 40, 155-156.

Angeli, V., Benassi, M.G., \& L’adavas, E. (2004b). Recovery of oculo-motor bias in neglect patients after prism adaptation. Neuropsychologia, 42(9), 1223-1234.

Antal, A., Kincses, T.Z., Nitsche, M.A., Bartfai, O., \& Paulus, W. (2004). Excitability changes induced in the human primary visual cortex by transcranial direct current stimulation: Direct electrophysiological evidence. Invest Ophthalmol Vis Sci, 45(2), 702-707.

Ardolino, G., Bossi, B., Barbieri, S., \& Priori, A. (2005). Nonsynaptic mechanisms underlie the after-effects of cathodal transcutaneous direct current stimulation of the human brain. J Physiol, 568(2), 653-663.

Avenanti, A., Annela, L., \& Serino, A. (2012a). Suppression of premotor cortex disrupts motor coding of peripersonal space.Neuroimage, 63(1), 281-288.

Avenanti, A., Coccia, M., Ladavas, E., Provinciali, L., \& Ceravolo, M.G. (2012b). Low-frequency rTMS promotes usedependent motor plasticity in chronic stroke A randomized trial. Neurol, 78(4), 256-264.

Ayache, S.S., Farhat, W.H., Zouari, H.G., Hosseini, H., Mylius, V., \& Lefaucheur, J.P. (2012). Stroke rehabilitation using noninvasive cortical stimulation: Motor deficit. Expert Rev Neurother, 12(8), 949-972.

Baker, J.M., Rorden, C.,\&Fridriksson, J. (2010). Using transcranial direct-current stimulation to treat stroke patients with aphasia. Stroke, 41(6), 1229-1236.

Barrett, A.M., Goedert, K.M., \& Basso, J.C. (2012). Prism adaptation for spatial neglect after stroke: Translational practice gaps. Nat Rev Neurol, 8(10), 567-577.

Batsikadze, G., Moliadze, V., Paulus, W., Kuo, M.F., \& Nitsche, M.A. (2013). Partially non-linear stimulation intensitydependent effects of direct current stimulation on motor cortex excitability in humans. J Physiol, 591(7), 19872000.

Boggio, P.S., Nunes, A., Rigonatti, S.P., Nitsche, M.A., Pascual-Leone, A., \& Fregni, F. (2007). Repeated sessions of noninvasive brain DC stimulation is associated with motor function improvement in stroke patients. Rest Neurol Neurosci, 25(2), 123-129.

Bolognini, N., Vallar, G., Casati, C., Latif, L.A., El-Nazer, R., Williams, J., Banco, E., Macea, D.D., Tesio, L., Chessa, C., \& Fregni, F. (2011). Neurophysiological and behavioral effects of tDCS combined with constraint-induced movement therapy in poststroke patients. Neurorehabil Neural Repair, 25(9), 819-829.

Bolognini, N., Olgiati, E., Maravita, A., Ferraro, F., \& Fregni, F. (2013). Motor and parietal cortex stimulation for phantom limb pain and sensations. Pain, 154(8), 1274-1280.

Bolognini, N., Convento, S., Banco, E., Mattioli, F., Tesio, L.,\&Vallar,G. (2015). Improving ideomotor limb apraxia by electrical stimulation of the left posterior parietal cortex. Brain, 138(2), 428-439.

Bowen A, Lincoln N. Cognitive rehabilitation for spatial neglect following stroke. Cochrane Database of Systematic Reviews 2007(2), Art. No.: CD003586. DOI: 10.1002/14651858.CD003 586.pub2

Brem, A.K., Unterburger, E., Speight, I., \& J"ancke, L. (2014). Treatment of visuospatial neglect with biparietal tDCS and cognitive training: A single-case study. Front Syst Neurosci, 8, 180. doi:10.3389/fnsys.2014.00180

Brighina, F., Bisiach, E., Oliveri, M., Piazza, A., La Bua,V., Daniele, O., \& Fierro, B. (2003). 1Hz repetitive transcranial magnetic stimulation of the unaffected hemisphere ameliorates contralesional visuospatial neglect in humans. Neurosci Lett, 336(2), 131-133.

Cappa, S.F., Benke, T., Clarke, S., Rossi, B., Stemmer, B., \& Heugten, C.M. (2005). EFNS guidelines on cognitive rehabilitation: Report of an EFNS task force. Eur J Neurol, 12(9), 665-680.

Chokron, S., Dupierrix, E., Tabert, M., \& Bartolomeo, P. (2007).Experimental remission of unilateral spatial neglect. Neuropsychologia, 45(14), 3127-3148.

Convento, S., Bolognini, N., Fusaro, M., Lollo, F., \& Vallar, G. (2014). Neuromodulation of parietal and motor activity affects motor planning and execution. Cortex, 57, 51-59.

Desmurget, M.,Gr'ea, H., Grethe, J.S., Prablanc, C., Alexander, G.E., \& Grafton, S.T. (2001). Functional anatomy of nonvisual feedback loops during reaching: A positron emission tomography study. J Neurosci, 21(8), 2919-2928.

Duvernoy, O.,Malm, T., Thuomas, K.A ., Larsson, S.G.,\&Hansson, H.E. (1991). CT andMRevaluation of pericardial and retrosternal adhesions after cardiac surgery. J Comput Assist Tomogr, 15(4), 555-560. 
Farn`e, A., Rossetti, Y., Toniolo, S., \& L`adavas, E. (2002). Ameliorating neglect with prism adaptation: Visuo-manual and visuo-verbal measures. Neuropsychologia, 40(7), 718-729.

Feng,W., Bowden, M.G.,\&Kautz, S. (2013). Reviewof transcranial direct current stimulation in poststroke recovery. Top Stroke Rehabil, 20(1), 68-77.

Fierro, B., Brighina, F., Oliveri, M., Piazza, A., La Bua, V., Buffa, D.,\&Bisiach, E. (2000). Contralateral neglect induced by right posterior parietal rTMS in healthy subjects. Neuroreport, 11(7), 1519-1521.

Fierro, B., Brighina, F., Piazza, A., Oliveri, M., \& Bisiach, E. (2001). Timing of right parietal and frontal cortex activity in visuo-spatial perception: A TMS study in normal individuals. Neuroreport, 12(11), 2605-2607.

Fortis, P., Maravita, A., Gallucci, M., Ronchi, R., Grassi, E., Senna, I., Olgiati, E., Perucca, L., Banco, E., Posteraro, L., Tesio, L., \& Vallar, G. (2010). Rehabilitating patients with left spatial neglect by prism exposure during a visuomotor activity.Neuropsychology, 24(6), 681.

Frassinetti, F., Angeli, V., Meneghello, F., Avanzi, S., \& Làdavas

E. (2002). Long-lasting amelioration of visuospatial neglect by prism adaptation. Brain, 125(3), 608-623.

Fregni, F., Boggio, P.S., Mansur, C.G., Wagner, T., Ferreira, M.J., Lima, M.C., Rigonatti, S. P., Marcolin, M.A., Freedman, S.D., Nitsche, M.A.,\&Pascual-Leone, A. (2005). Transcranial direct current stimulation of the unaffected hemisphere in stroke patients. Neuroreport, 16(14), 1551-1555.

Fregni, F., Boggio, P.S., Valle, A.C., Rocha, R.R., Duarte, J., Ferreira, M.J., Wagner, T., Fecteau, S., Rigonatti, S.P., Riberto, M., Freedman, S.D., \& Pascual-Leone, A. (2006). A sham controlled trial of a 5-day course of repetitive transcranial magnetic stimulation of the unaffected hemisphere in stroke patients. Stroke, 37(8), 2115-2122.

Fridriksson, J., Richardson, J.D., Baker, J.M., \& Rorden, C. (2011). Transcranial direct current stimulation improves naming reaction time in fluent aphasia a double-blind, sham-controlled study. Stroke, 42(3), 819-821.

Gandiga, P.C., Hummel, F.C., \& Cohen, L.G. (2006). Transcranial DC stimulation (tDCS): A tool for double-blind sham-controlled clinical studies in brain stimulation. Clin Neurophysiol, 117(4), 845-850.

Giacobbe, V., Krebs, H.I., Volpe, B.T., Pascual-Leone, A., Rykman,A., Zeiarati, G., Fregni, F., Dipietro, L., Thickbroom, G.W., \& Edwards, D.J. (2013). Transcranial direct current stimulation (tDCS) and robotic practice in chronic stroke: The dimension of timing. NeuroRehabilitation, 33(1), 49-56.

Goedert, K.M., Boston, R.C.,\&Barrett, A.M. (2013). Advancing the science of spatial neglect rehabilitation: An improved statistical approach with mixed linear modeling. Front Hum Neurosci, 7, 211. doi:

10.3389/fnhum.2013.00211

Halligan, P.W., Marshall, J.C., \& Wade, D.T. (1989). Visuospatial neglect: Underlying factors and test sensitivity. Lancet, 334(8668), 908-911.

Horvath, J.C., Forte, J.D., \& Carter, O. (2015). Evidence that transcranial direct current stimulation (tDCS) generates little to-no reliable neurophysiologic effect beyond MEP amplitude modulation in healthy human subjects: A systematic review. Neuropsychologia, 66, 213-236.

Hummel, F.C., \& Cohen, L.G. (2006). Non-invasive brain stimulation: Anewstrategy to improve neurorehabilitation after stroke? Lancet Neurol, 5(8), 708-712.

Hummel, F.C., Celnik, P., Pascual-Leone, A., Fregni, F., Byblow, W.D., Buetefisch, C.M., Rothwell, J., Cohen, L.G., \& Gerloff, C. (2008). Controversy: Noninvasive and invasive cortical stimulation show efficacy in treating stroke patients. Brain Stimul, 1(4), 370-382.

Jacobson, L., Koslowsky, M., \& Lavidor, M. (2012). tDCS polarity effects in motor and cognitive domains: A metaanalytical review. Exp Brain Res, 216(1), 1-10.

Khedr, E.M., Ahmed, M.A., Fathy, N., \& Rothwell, J.C. (2005). Therapeutic trial of repetitive transcranial magnetic stimulation after acute ischemic stroke. Neurol, 65(3),466-468.

Khedr, E.M., Abdel-Fadeil, M.R., Farghali, A., \& Qaid, M. (2009). Role of 1 and 3Hz repetitive transcranial magnetic stimulation on motor function recovery after acute ischaemic stroke. Eur J Neurol, 16(12), 1323-1330.

Kim, Y.H., You, S.H., Ko, M.H., Park, J.W., Lee, K.H., Jang, S.H., Yoo, W.K., \& Hallett, M. (2006). Repetitive transcranial magnetic stimulation-induced corticomotor excitability and associated motor skill acquisition in chronic stroke. Stroke, 37(6), 1471-1476.

Kim, D.Y., Lim, J.Y., Kang, E.K., You, D.S., Oh, M.K., Oh, B.M., \& Paik, N.J. (2010). Effect of transcranial direct current stimulation on motor recovery in patients with subacute stroke. Am J Phys Med Rehabil, 89(11), 879-886. Kinsbourne, M. (1993). Orientational bias model of unilateral neglect: Evidence from attentional gradients within hemispace, In John Marshall \& Ian Robertson (eds.), Unilateral Neglect:Clinical And Experimental Studies (Brain Damage, Behaviour and Cognition). Psychology Press, pp. 63-86.

Ko, M.H., Han, S.H., Park, S.H., Seo, J.H., \& Kim, Y.H. (2008). Improvement of visual scanning after DC brain polarization of parietal cortex in stroke patients with spatial neglect. Neurosci Lett, 448(2), 171-174.

Koch, G., Oliveri, M., Cheeran, B., Ruge, D., Gerfo, E.L., Salerno, S., Torriero, S., Marconi, B., Mori, F., Driver, J., Rothwell, J.C., \& Caltagirone, C. (2008). Hyperexcitability of parietal-motor functional connections in the intact lefthemisphere of patients with neglect. Brain, 131(12), 3147-3155.

Koch, G., Bonni, S., Giacobbe, V., Bucchi, G., Basile, B., Lupo, F., Versace, V., Bozzali, M., \& Caltagirone, C. (2012). Thetaburst stimulation of the left hemisphere accelerates recovery of hemispatial neglect. Neurol, 78(1), 24-30. 
Koganemaru, S., Mima, T., Thabit, M.N., Ikkaku, T., Shimada, K., Kanematsu, M., Takahashi, K., Fawi, G., Takahashi, R., Fukuyama, H., \& Domen, K. (2010). Recovery of upper-limb function due to enhanced use-dependent plasticity in chronic stroke patients. Brain, 133(11), 3373-3384.

L`adavas, E., Bonifazi, S., Catena, L., \& Serino, A. (2011). Neglect rehabilitation by prism adaptation: Different procedures have different impacts. Neuropsychologia, 49(5), 1136-1145.

Liebetanz, D., Nitsche, M.A., Tergau, F., \& Paulus, W. (2002). Pharmacological approach to the mechanisms of transcranial DC-stimulation-induced after-effects of human motor cortex excitability. Brain, 125(10), $2238-2247$. Lim, J.Y., Kang, E.K., \& Paik, N.J. (2010). Repetitive transcranial magnetic stimulation for hemispatial neglect in patients after stroke: An open-label pilot study. J Rehabil Med, 42(5), 447-452.

Lindenberg, R., Renga,V., Zhu, L.L., Nair, D.,\&Schlaug, G. (2010). Bihemispheric brain stimulation facilitates motor recovery in chronic stroke patients. Neurology, 75(24), 2176-2184.

Loo, C.K., Alonzo, A., Martin, D., Mitchell, P.B., Galvez, V., \& Sachdev, P. (2012). Transcranial direct current stimulation for depression: 3-week, randomised, sham-controlled trial. Br J Psychiatry, 200(1), 52-59.

Loo, C.K., Sachdev, P., Martin, D., Pigot, M., Alonzo, A., Malhi, G.S., Lagopoulos, J., \& Mitchell, P. (2010). A double-blind, sham-controlled trial of transcranial direct current stimulation for the treatment of depression. Int $\mathrm{J}$ Neuropsychopharmacol, 13(1), 61-69.

Luaut'e, J., Halligan, P., Rode, G., Rossetti,Y.,\&Boisson, D. (2006a). Visuo-spatial neglect: A systematic review of current interventions and their effectiveness. Neurosci Biobehav Rev, 30(7), 961-982.

Luaut'e, J., Michel, C., Rode, G., Pisella, L., Jacquin-Courtois, S.,Costes, N., Cotton, F., le Bars, D., Boisson, D., Halligan, P., \& Rossetti, Y. (2006b). Functional anatomy of the therapeutic effects of prism adaptation on left neglect. Neurol, 66(12),

1859-1867.

Luaut'e, J., Schwartz, S., Rossetti, Y., Spiridon, M., Rode, G., Boisson, D., \& Vuilleumier, P. (2009). Dynamic changes in brain activity during prism adaptation. J Neurosci, 29(1), 169-178.

Mansur, C.G., Fregni, F., Boggio, P.S., Riberto, M., Gallucci-Neto, J., Santos, C.M., Wagner, T., Rigonatti, S.P., Marcolin, M.A. \& Pascual-Leone, A. (2005). A sham stimulation-controlled trial of rTMS of the unaffected hemisphere in stroke patients. Neurol, 64(10), 1802-1804.

Marangolo, P., Marinelli, C.V., Bonifazi, S., Fiori, V., Ceravolo, M.G., Provinciali, L., \& Tomaiuolo, F. (2011). Electrical stimulation over the left inferior frontal gyrus (IFG) determines long-term effects in the recovery of speech apraxia in three chronic aphasics. Behav Brain Res, 225(2), 498-504.

Minamoto, T., Azuma, M., Yaoi, K., Ashizuka, A., Mima, T., Osaka, M., Fukuyama, H., Osaka, N. (2014). The anodal tDCS over the left posterior parietal cortex enhances attention toward a focus word in a sentence. Front Hum Neurosci, 8, 992. doi:10.3389/fnhum.2014.00992

Moliadze, V., Antal, A., \& Paulus, W. (2010). Electrode-distance dependent after-effects of transcranial direct and random noise stimulation with extracephalic reference electrodes. Clinical Neurophysiology, 121(12), $2165-2171$. Mylius, V., Ayache, S.S., Zouari, H.G., Aoun-Seba“1ti, M., Farhat, W.H., \& Lefaucheur, J.P. (2012). Stroke rehabilitation using noninvasive cortical stimulation: Hemispatial neglect. Expert Review of Neurotherapeuthics, 12(8), 983-991.

Neggers, S.F., \& Bekkering, H. (2000). Ocular gaze is anchored to the target of an ongoing pointing movement. Journal of Neurophysiology, 83(2), 639-651.

Nitsche, M.A., \& Paulus, W. (2000). Excitability changes induced in the human motor cortex by weak transcranial direct current stimulation. The Journal of Physiology, 527(3), 633-639.

Nitsche, M.A., \& Paulus, W. (2001). Sustained excitability elevations induced by transcranial DC motor cortex stimulation in humans. Neurology, 57(10), 1899-1901.

Nitsche, M.A., Liebetanz, D., Antal, A., Lang, N., Tergau, F., \& Paulus, W. (2003a). Modulation of cortical excitability by weak direct current stimulation-technical, safety and functional aspects. Suppl Clin Neurophysiol, 56(3), 255-276.

Nitsche, M.A., Nitsche, M.S., Klein, C.C., Tergau, F., Rothwell, J.C., \& Paulus, W. (2003b). Level of action of cathodal DC polarisation induced inhibition of the human motor cortex. Clin Neurophysiol, 114(4), 600-604.

Nitsche, M.A., Cohen, L.G., Wassermann, E.M., Priori, A., Lang, N., Antal, A., Paulus,W., Hummel, F., Boggio, P.S., Fregni, F., \& Pascual-Leone, A.(2008). Transcranial direct current stimulation: State of the art 2008. Brain Stimul, 1(3), 206-223.

Nyffeler, T., Cazzoli, D., Hess, C.W.,\&M“uri, R.M. (2009). One session of repeated parietal theta burst stimulation trains induces long-lasting improvement of visual neglect. Stroke, 40(8), 2791-2796.

Nys, G.M.S., De Haan, E.H.F., Kunneman, A., De Kort, P.L.M., \& Dijkerman, H.C. (2008). Acute neglect rehabilitation using repetitive prism adaptation: A randomized placebo-controlled trial. Rest Neurol Neurosci, 26(1), 112.

O’Connell, N.E., Cossar, J., Marston, L., Wand, B.M., Bunce, D., Moseley, G.L., \& De Souza, L.H. (2012). Rethinking clinical trials of transcranial direct current stimulation: Participant and assessor blinding is inadequate at intensities of 2mA. PLoS One, 7(10), e47514.

Oliveri, M., Bisiach, E., Brighina, F., Piazza, A., La Bua, V., Buffa, D., \& Fierro, B. (2001). rTMS of the unaffected hemisphere transiently reduces contralesional visuospatial hemineglect. Neurol, 57(7), 1338-1340. 
Poreisz, C., Boros, K., Antal, A.,\&Paulus,W. (2007). Safety aspects of transcranial direct current stimulation concerning healthy subjects and patients. Brain Res Bull, 72(4), 208-214.

Redding, G.M., \& Wallace, B. (2006). Prism adaptation and unilateral neglect: Review and analysis. Neuropsychologia, 44(1),1-20.

Rorden, C., \& Brett, M. (2000). Stereotaxic display of brain lesions.Behav Neurol, 12(4), 191-200.

Rossetti, Y., Rode, G., Pisella, L., Farn`e, A., Li, L., Boisson, D., \& Perenin, M.T. (1998). Prism adaptation to a rightward optical deviation rehabilitates left hemispatial neglect. Nature, 395(6698), 166-169.

Saj, A., Cojan, Y., Vocat, R., Luaut'e, J., \& Vuilleumier, P. (2013). Prism adaptation enhances activity of intact frontoparietal areas in both hemispheres in neglect patients. Cortex, 49(1), 107-119.

Serino, A., Angeli, V., Frassinetti, F., \& Làdavas, E. (2006). Mechanisms underlying neglect recovery after prism adaptation. Neuropsychologia, 44(7), 1068-1078.

Serino, A., Bonifazi, S., Pierfederici, L., \& L`adavas, E. (2007). Neglect treatment by prism adaptation: What recovers and for how long. Neuropsychol Rehabil, 17(6), 657-687.

Serino, A., Barbiani, M., Rinaldesi, M.L., \& L`adavas, E. (2009). Effectiveness of prism adaptation in neglect rehabilitation a controlled trial study. Stroke, 40(4), 1392-1398.

Shindo, K., Sugiyama, K., Huabao, L., Nishijima, K., Kondo, T., \& Izumi, S. (2006). Long-term effect of lowfrequency repetitive transcranial magnetic stimulation over the unaffected posterior parietal cortex in patients with unilateral spatial neglect. J Rehabil Med, 38(1), 65-67.

Song,W., Du, B., Xu, Q., Hu, J.,Wang, M., \& Luo, Y. (2009). Lowfrequency transcranial magnetic stimulation for visual spatial neglect: A pilot study. J Rehabil Med, 41(3), 162-165.

Sparing, R., Thimm, M., Hesse, M.D., K“ust, J., Karbe, H., \& Fink, G.R. (2009). Bidirectional alterations of interhemispheric parietal balance by non-invasive cortical stimulation. Brain, 132(11), 3011-3020.

Sprague, J.M. (1966). Interaction of cortex and superior colliculus in mediation of visually guided behavior in the cat. Science, 153(3743), 1544-1547.

Stagg, C.J., \& Nitsche, M.A. (2011). Physiological basis of transcranial direct current stimulation. Neuroscientist, 17(1), 37-53.

Sunwoo, H., Kim,Y.H., Chang,W.H., Noh, S., Kim, E.J.,\&Ko, M.H. (2013). Effects of dual transcranial direct current stimulation on post-stroke unilateral visuospatial neglect. Neurosci Lett, 554, 94-98.

Takeuchi, N., Chuma, T., Matsuo, Y., Watanabe, I., \& Ikoma, K. (2005). Repetitive transcranial magnetic stimulation of contralesional primary motor cortex improves hand function after stroke. Stroke, 36(12), 2681-2686.

Talelli, P., \& Rothwell, J. (2006). Does brain stimulation after stroke have a future? Curr Opin Neurol, 19(6), 543-550. Tremblay, S., Beaule, V., Lepage, J.F.,\&Théoret, H. (2013). Anodal transcranial direct current stimulation modulates GABAB-related intracortical inhibition in the M1 of healthy individuals. Neuroreport, 24(1), 46-50.

Turton, A.J., O’Leary, K., Gabb, J.,Woodward, R., \& Gilchrist, I.D.(2010). A single blinded randomised controlled pilot trial of prism adaptation for improving self-care in stroke patients with neglect. Neuropsychol Rehabil, 20(2), 180196.

Tzourio-Mazoyer, N., Landeau, B., Papathanassiou, D., Crivello, F., Etard, O., Delcroix, N., Mazoyer, B., \& Joliot, M. (2002). Automated anatomical labeling of activations in SPM using a macroscopic anatomical parcellation of the MNI MRI singlesubject brain. Neuroimage, 15(1), 273-289.

Vangkilde, S., \& Habekost, T. (2010). Finding Wally: Prism adaptation improves visual search in chronic neglect. Neuropsychologia, 48(7), 1994-2004.

Wilson, B., Cockburn, J., \& Halligan, P. (1987). Development of a behavioral test of visuospatial neglect. Arch Phys Med Rehabil, 68(2), 98-102.

Zoccolotti, P., Cantagallo, A., De Luca, M., Guariglia, C., Serino, A., \& Trojano, L. (2011). Selective and integrated rehabilitation programs for disturbances of visual/spatial attention and executive function after brain damage: A neuropsychological evidence-based review. Eur J Phys Rehabil Med, 47(1), 123-147. 
Table 1

Demographic and Clinical Data for Patients included in the c-tDCS, the a-tDCS and Sham groups

\begin{tabular}{|c|c|c|c|c|c|}
\hline Case No. & Gender & Age & Education & $\begin{array}{l}\text { Months } \\
\text { from illness }\end{array}$ & $\begin{array}{l}\text { BIT conventional } \\
\text { at admission }\end{array}$ \\
\hline c-tDCS P1 & $\mathrm{M}$ & 59 & 18 & 3 & 128 \\
\hline c-tDCS P2 & $\mathrm{F}$ & 80 & 4 & 2 & 105 \\
\hline c-tDCS P3 & M & 73 & 3 & 3 & 33 \\
\hline c-tDCS P4 & $\mathrm{F}$ & 69 & 5 & 2 & 123 \\
\hline c-tDCS P5 & $\mathrm{F}$ & 78 & 3 & 2 & 129 \\
\hline c-tDCS P6 & M & 71 & 5 & 2 & 127 \\
\hline c-tDCS P7 & M & 74 & 5 & 2 & 65 \\
\hline c-tDCS P8 & $\mathrm{F}$ & 70 & 8 & 2 & 79 \\
\hline Mean & & 72 & 6.4 & 2.2 & 98 \\
\hline a-tDCS P1 & $\mathrm{F}$ & 78 & 5 & 4 & 122 \\
\hline a-tDCS P2 & M & 60 & 16 & 8 & 60 \\
\hline a-tDCS P3 & $\mathrm{F}$ & 77 & 5 & 4 & 109 \\
\hline a-tDCS P4 & $\mathrm{F}$ & 46 & 18 & 3 & 120 \\
\hline a-tDCS P5 & M & 62 & 18 & 3 & 123 \\
\hline a-tDCS P6 & M & 73 & 5 & 3 & 86 \\
\hline a-tDCS P7 & $\mathrm{F}$ & 67 & 5 & 3 & 116 \\
\hline a-tDCS P8 & M & 71 & 6 & 3 & 92 \\
\hline a-tDCS P9 & $\mathrm{F}$ & 73 & 3 & 2 & 106 \\
\hline a-tDCS P10 & $\mathrm{M}$ & 62 & 18 & 2 & 45 \\
\hline a-tDCS P11 & M & 57 & 7 & 2 & 127 \\
\hline Mean & & 66 & 9.6 & 3.3 & 101 \\
\hline Sham P1 & $\mathrm{F}$ & 70 & 3 & 2 & 41 \\
\hline Sham P2 & $\mathrm{M}$ & 49 & 13 & 6 & 95 \\
\hline Sham P3 & $\mathrm{F}$ & 74 & 5 & 2 & 109 \\
\hline Sham P4 & M & 63 & 18 & 2 & 121 \\
\hline Sham P5 & $\mathrm{F}$ & 72 & 8 & 3 & 120 \\
\hline Sham P6 & M & 70 & 8 & 8 & 128 \\
\hline Sham P7 & M & 69 & 13 & 3 & 109 \\
\hline Sham P8 & M & 59 & 8 & 2 & 125 \\
\hline Sham P9 & $\mathrm{F}$ & 70 & 5 & 2 & 127 \\
\hline Sham P10 & $\mathrm{F}$ & 78 & 5 & 3 & 123 \\
\hline Sham P11 & M & 65 & 5 & 2 & 35 \\
\hline Mean & & 67 & 8.2 & 3.2 & 103 \\
\hline
\end{tabular}




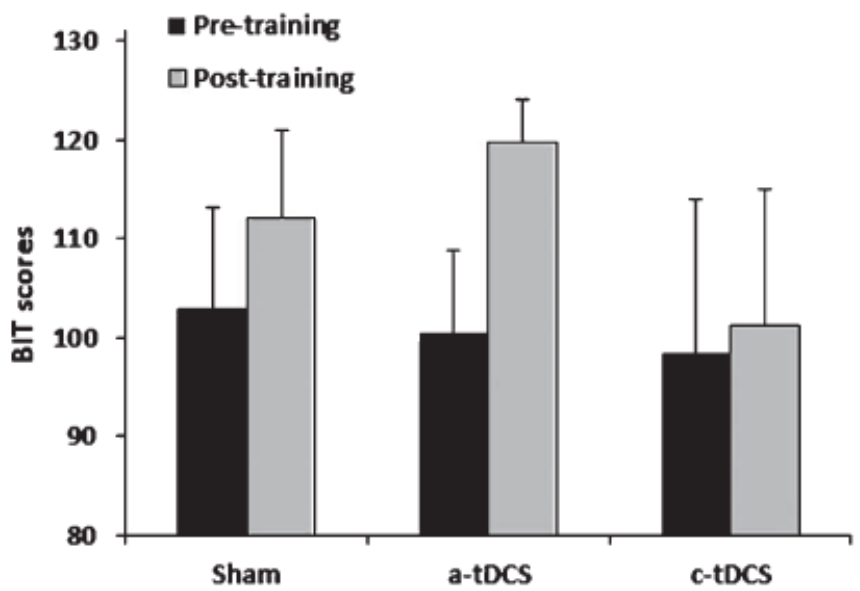

Fig. 1. Effects of combined tDCS and PA treatment. Mean (and S.E.M.) scores at the BIT scale obtained before and after the treatment by patients submitted to PA training plus Sham tDCS, a-tDCS, and c-tDCS.

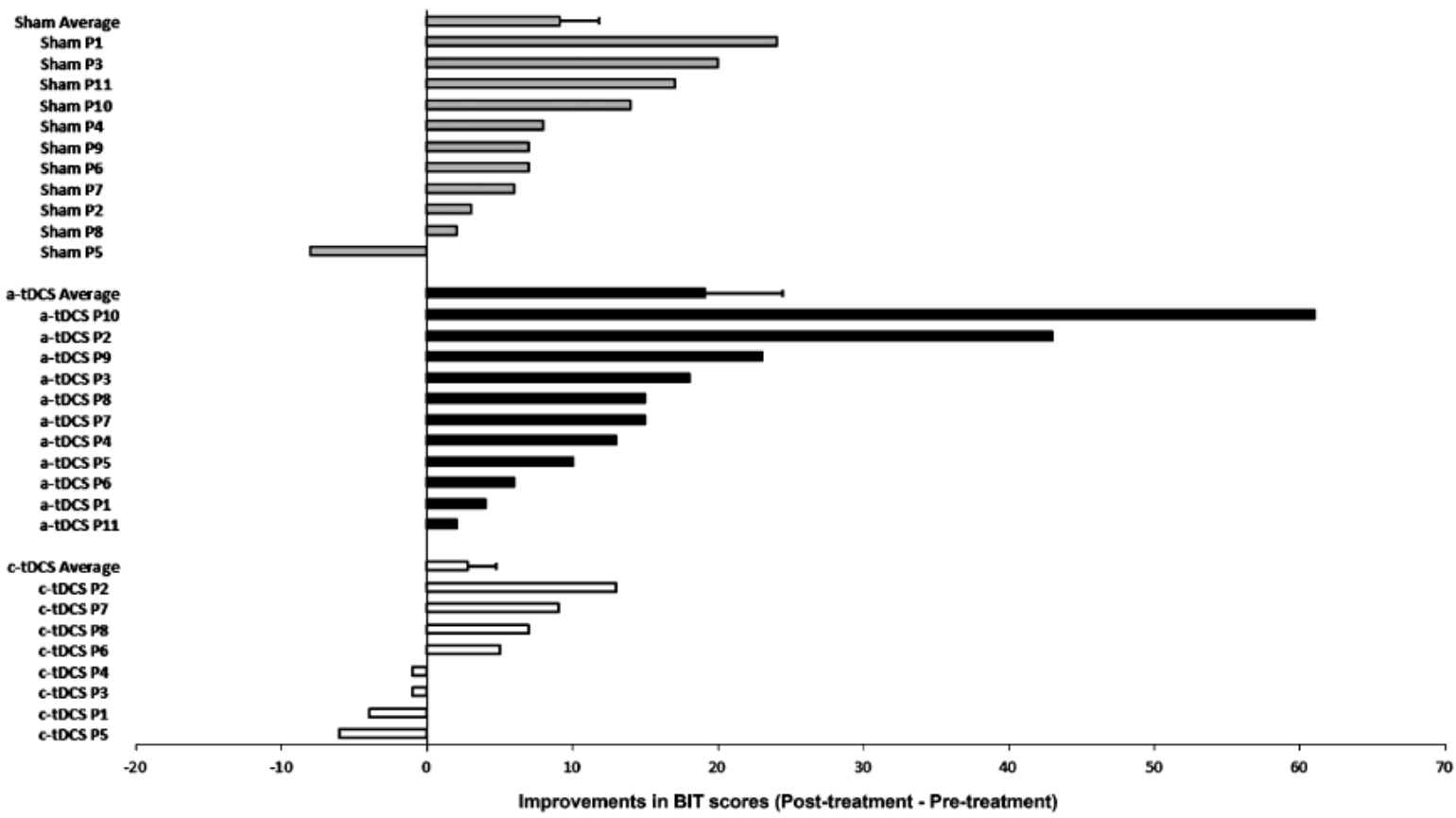

Fig. 2. Improvements in BIT scores by individual patients from the Sham, a-tDCS and c-tDCS groups. Scores are computed as the difference between BIT scores obtained after the treatment and before the treatment. Error bars represent S.E.M. 


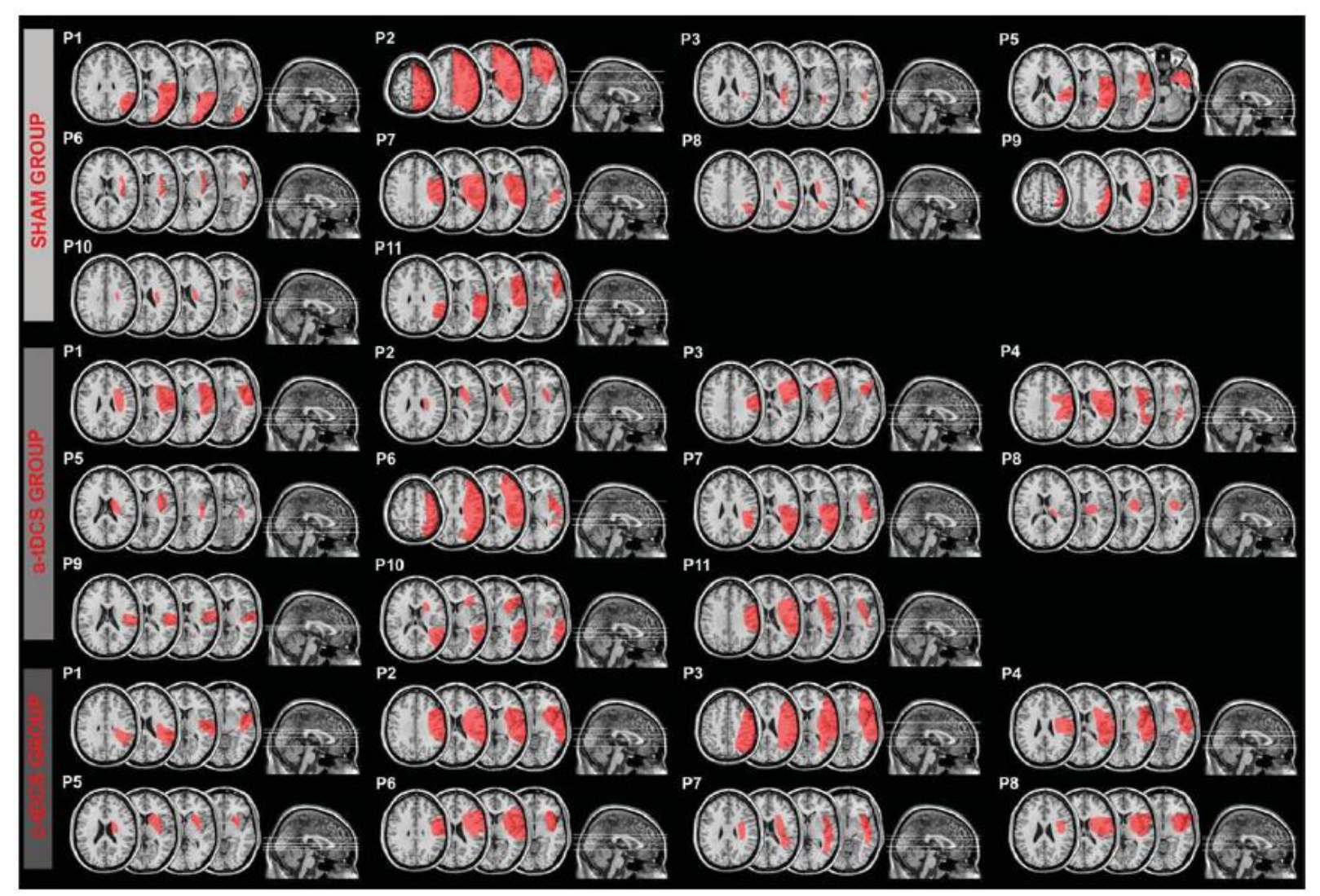

Fig. 3. Lesion reconstruction images from MRI, reported onto the normalized MNI template for patients submitted to PA training plus Sham tDCS, a-tDCS and c-tDCS. 